\title{
Physiological Tremor Filtering without Phase Distortion for Robotic Microsurgery
}

\author{
Kabita Adhikari*, Sivanagaraja Tatinati, Member, IEEE, Kalyana C. Veluvolu, Senior Member, IEEE, and \\ Jonathon A. Chambers, Fellow, IEEE
}

\begin{abstract}
All existing physiological tremor filtering algorithms, developed for robotic microsurgery, use non-linear phase pre-filters to isolate the tremor signal. Such filters cause phase distortion to the filtered tremor signal and limit the filtering accuracy. We revisited this long-standing problem to enable filtering of the physiological tremor without any phase distortion. We developed a combined estimation-prediction paradigm that offers zero-phase type filtering. The estimation is achieved with the mathematically modified recursive singular spectrum analysis algorithm and the prediction is delivered with the standard extreme learning machine. In addition, to limit the computational cost; we developed two moving window versions of this structure, appropriate for real-time implementation. The proposed paradigm preserved the natural phase of the filtered tremor. It achieved the key performance index of error limitation below $10 \mu \mathrm{m}$, yielding the estimation accuracy larger than $70 \%$, at a time delay of $36 \mathrm{~ms}$ only. Both moving window versions of the proposed approach restricted the computational cost considerably; whilst offering the same performance. It is for the first time that effective estimation of the physiological tremor is achieved, without any pre-filtering and phase distortion. This proposed method is feasible for real-time implantation. Clinical translation of the proposed paradigm can significantly enhance the outcome in hand-held surgical robotics.
\end{abstract}

Note to Practitioners - The imprecision caused by physiological hand tremor in microsurgeries has motivated researchers to innovate an efficient tremor compensating technique that can improve the surgical performance. Yet, all the existing tremor filtering algorithms, implemented in hand-held surgical instruments, use non-linear phase pre-filters to separate the tremor signal. The inherent phase distortion caused by such pre-filters restricts the filtering performance significantly and renders the existing methods inadequate for hand-held robotic surgery. Motivated by this, we proposed a novel estimator-predictor based framework, by adopting the modified recursive singular spectrum analysis estimator and the extreme learning machine predictor. The proposed framework filters the tremor signal accurately, without distorting it, but at a small fixed lag. In a set of rigorous testing performed by emulating a real-time processing, the proposed

Manuscript received August 29, 2019. This work is supported by Newcastle University, Newcastle NE1 7RU, UK. The research was also supported in part by the National Research Foundation (NRF) of Korea through the Ministry of Education, Science and Technology under Grant NRF2018R1A6A1A03025109.

${ }^{*} \mathrm{~K}$. Adhikari is with the School of Engineering, Newcastle University, UK (Correspondence e-mail: kabita.adhikari@newcastle.ac.uk.)

S. Tatinati is with the School of Electrical and Electronic Engineering, Nanyang Technological University, Singapore.

K. C. Veluvolu is with the School of Electronics Engineering, Kyungpook National University, South Korea.

J. A. Chambers is with the School of Engineering, Newcastle University, UK.

The MATLAB implementation codes of the RSSA-ELM algorithms supporting this publication will be openly available at DOI: 10.25405/data.ncl.13280501. Please contact Newcastle Research Data Service at rdm@ncl.ac.uk for access instructions. algorithm showed higher performance compared to the state-ofthe-art algorithms. This validates not only its suitability for realtime implantation but also its potential to improve the surgical performance, which has been limited by the distorted filtering. Nonetheless, we have presented a proof-of-principle framework for a distortion free filtering, but its full implementation in a real surgical instrument, such as Micron or ITrem, requires a substantial amount of experimental testing and verification. It can be also applicable in a wide range of areas, including healthcare, digital manufacturing, smart automation and control, and various other robotic technologies where efficient filtering of advanced sensors data is highly desirable. In future, we will develop the multidimensional model of the proposed framework to enable filtering of tremor in the $x y z$ axes simultaneously.

Index Terms-Extreme learning machine, physiological tremor, recursive singular spectrum analysis, robotic microsurgery.

\section{INTRODUCTION}

$\mathbf{P}$ HYSIOLOGICAL tremor is an involuntary and quasiperiodic movement that commonly exists in normal human hand motion [1]. Its frequency spectrum lies in the range of $6-14 \mathrm{~Hz}$ [2], [3] and its magnitude can be as high as $100 \mu \mathrm{m} \mathrm{[4]-[7].} \mathrm{This} \mathrm{level} \mathrm{of} \mathrm{oscillation} \mathrm{is} \mathrm{not} \mathrm{so} \mathrm{much} \mathrm{of}$ a problem for day-to-day tasks but causes detrimental effects in microsurgery that requires precise hand positioning [8], [9]. The undesired vibration of the tip of a surgical tool, caused by natural hand tremor degrades the efficacy of microsurgical interventions, such as treatment procedures for retinal vascular occlusions or peeling of thin membranes, in which a positioning accuracy of $10 \mu \mathrm{m}$, or less, is typically required [10]-[12].

The movement of the tip of a microsurgery tool comprises voluntary motion and involuntary motion that includes the physiological hand tremor, jerk and low frequency drift. Amongst all, the physiological tremor has most adverse effect on surgical micromanipulation as jerk occurs rarely and drift can be adjusted by visual feedback [13]. To attenuate the physiological hand tremor, there have been remarkable advancements in surgical robotics in last three decades, including tele-robotic and robot-assisted systems [14]-[16], steady-hand robotic systems [17], [18] and smart hand-held surgical devices [2], [3], [19], [20]. Amongst all, direct hand surgery still retains its appeal as it provides surgeons'expertise and natural feel; and is intuitive due to the fast correspondence between the visual feedback and voluntary hand motion of the surgeon who is directly holding the instrument [2], [6], [16], [21]. Despite these advantages, the high level of hand positioning and dexterity required for microsurgery restricts a number of qualified surgeons due to their natural tremor [19]. Hence to 
enhance the dexterity of direct hand surgery, smart hand-held surgical instruments such as Micron [2], [19] and ITrem [20] have been developed with the aim to effectively alleviate the physiological tremor from the tip of the instrument.

The voluntary motion of the hand is confined below $4 \mathrm{~Hz}$ [22]. Hence, initially in human-machine interface, linear-phase lowpass or bandstop filters were applied directly to the whole motion to suppress the tremor [23], [24]. To be effective, these filters require a large filter order that introduces a considerable time delay [25]. Therefore the use of these filters was discouraged [5], [26]. Riviere et al. [2] proposed a smart hand-held device, called Micron, which senses its own motion, isolates the tremor from other components, then actuates the tip in an equal but opposite direction to cancel the tremor effectively. For effective performance of Micron, the accurate estimation of the tremor is crucial otherwise exact anti-phase actuation cannot be accomplished. The amplitude and the spectrum of the tremor are however unknown and time-varying. Hence, to track the non-stationary tremor, they utilised an adaptive filtering approach called a weighted Fourier linear combiner (wFLC) [6]. In recent years, wFLC has been extended to autoregressive [27], bandlimited [28]-[30] and quaternion [31] versions. To function accurately, all of these adaptive techniques required a reference tremor signal, which was generated by pre-filtering the whole motion with an infinite impulse response (IIR) filter.

\section{A. The detrimental effect of pre-filtering}

The key shortcoming of the wFLC algorithm is caused by the aforementioned pre-filter. The time delay introduced by such IIR pre-filters is much less compared to the delay introduced by linear phase filters but they cause frequency selective delays. Fig. 1A shows the time delay versus frequency plot for the $5^{\text {th }}$-order bandpass Butterworth IIR filter with a passband of $2-20 \mathrm{~Hz}$, which was used in [27], [30], [32]. In addition, it shows the same for a linear phase Hamming-window based finite impulse response (FIR) filter with order 1000. The IIR filter causes a frequency-specific time delay. However, the FIR filter features a constant time delay of $2 \mathrm{~s}\left(=\frac{1000}{2} \times 4 \mathrm{~ms}\right.$; sampling time is $4 \mathrm{~ms}$ ) to all the frequencies in its passband. A time-domain representation of the frequency-specific effect of the above IIR pre-filter on four tones in the tremor frequency band is shown in Fig. 1B.

Fig. 1C shows a $2 \mathrm{~s}$ plot of a real tremor signal (black) obtained for a novice subject by using a zero-phase pre-filter with the same specifications as the IIR pre-filters of Fig. 1A. The IIR pre-filter completely distorts the shape of the reference tremor signal. The FIR pre-filter accurately approximates the tremor signal; however, the trade-off is extremely high delay which makes it inapplicable for real-time implementation. The resulting frequency-specific distortion of the IIR prefilter limits the efficacy of the wFLC algorithm in real-time implementations. All of the off-springs of the wFLC, e.g. [28], [31], [32], inherit this shortcoming.

To overcome this frequency-specific distortion effect, Veluvolu et al. [30] proposed a multistep prediction method with a fixed-horizon. They postulated that if the exact delay caused

\section{Effect of Pre-filtering}

A Frequency-dependent time delay

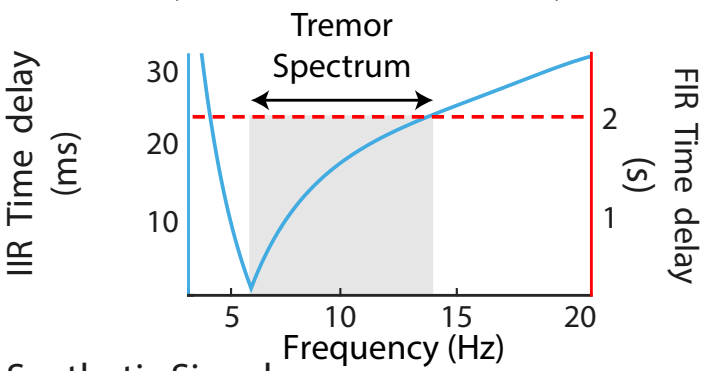

B Synthetic Signal

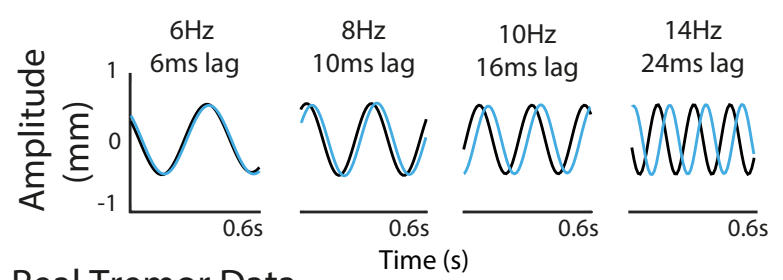

C Real Tremor Data

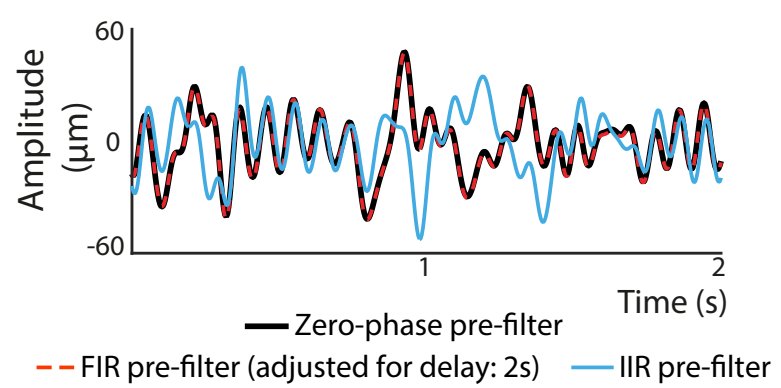

Fig. 1. The effect of pre-filtering: (A) Time versus frequency responses of the linear-phase FIR and non-linear phase IIR pre-filters; (B) Frequency-specific phase delay caused by the IIR filter introduced in A. (C) The reference tremor signal, of a novice surgeon, generated with zero-phase, IIR, and FIR pre-filters. Unlike the FIR filter the IIR pre-filter distorts the shape of the tremor signal. The output of the FIR filter is adjusted for the 2 s delay.

by a non-linear phase filter is pre-known, then it can be overcome by using multistep prediction. However, the frequency selective delay experienced by the reference tremor cannot be pre-known in real-time, as the spectrum of the tremor signal is non-stationary. This was later acknowledged in [32], [33]. The authors agreed that the limitation of the conventional non-linear phase filters is their inherent phase distortion. As a solution, they proposed a phase correction technique based on the moving window least squares support vector machines (MWLSSVM) and the one-dimensional online sequential robust ELM (1D-OSRELM) algorithms, which yielded better filtering performance [32], [33]. These algorithms however still relied on IIR pre-filters to isolate the tremor signal from the whole motion. As a consequence, the isolated tremor was completely distorted. The distorted tremor signal was then rectified by mapping it with the non-distorted tremor signal obtained from a zero-phase filtering. Since the tremor signal is non-stationary, for an accurate rectification, these methods necessitated the tremor signal to be zero-phase filtered continuously to adapt to its changing characteristics. However, the zero-phase filtering is a non-causal system and 
cannot be performed in real-time. This limitation renders the aforementioned methods inadequate for a real-time implementation. Although, frequent offline mapping can be performed, the filter's transient characteristic restricts the prompt update of the mapping, which degrades the filtering performance significantly.

In this paper, we offer a new paradigm to address this problem. Instead of pre-filtering the tremor signal, we directly extract the voluntary and the tremor signal from the whole motion by decomposing it. We first extract the voluntary signal and then deduct it from the whole motion to approximate the tremor signal. Our approach comprises two units: 1) an estimator and 2) a predictor. We achieve zero-phase type estimation of voluntary motion by adopting the recursive singular spectrum analysis (RSSA) [34] algorithm. However, this zero-phase estimation comes at the cost of a fixed time delay. To shorten this delay, we append a predictor unit, that is an extreme learning machine (ELM) [35] to the RSSA estimator. This combined structure is tested with the real physiological tremor data recorded from five novices and four microsurgeons, and the results show that the proposed paradigm: 1) yields zero-phase type filtering of the voluntary and tremor signals without causing any distortion; and 2) attenuates the unwanted tremor below $10 \mu \mathrm{m}$ limit which is desired in microsurgeries. The main contribution of this paper is the novel paradigm that can filter the tremor signal as accurately as a high order linear phase filter, yet within a small processing delay like a non-linear phase filter. Hence it offers the best of both conventional filters, without need of any prefiltering or zero-phase filtering. This is the major advantage of the proposed framework compared to the existing state-ofthe-art techniques.

The paper is organised as follows. Section II provides the theoretical overviews of the SSA and ELM algorithms. In Section III, we present the proposed RSSA-ELM method, and moving window RSSA-ELM methods. We evaluate offline and real-time performance of these algorithms using real tremor data in Section IV. Section V contains discussion of results and finally, we conclude in Section VI. Preliminary results were reported in [36].

\section{Review of Existing Methods}

\section{A. Singular Spectrum Analysis}

The SSA algorithm decomposes a time series into a number of interpretable components by using singular value decomposition (SVD) of a lag-covariance matrix formed from a time series. The SSA algorithm is computed in two complementary stages: decomposition and reconstruction [34].

1) Decomposition: The decomposition stage comprises two substages, embedding followed by SVD. In the embedding substage, the original one dimensional time series $\mathbf{x}_{N}=$ $\left[x_{1}, x_{2}, \cdots, x_{N}\right]$ is reconstructed into a multidimensional series. The multidimensional series is a sequence of $L$-lagged vectors $\mathbf{x}_{i}$, which forms the trajectory matrix, $\mathbf{X}_{L \times K}=\left[\begin{array}{lllll}\mathbf{x}_{1} & \mathbf{x}_{2} & \mathbf{x}_{3} & \cdots & \mathbf{x}_{K}\end{array}\right]$ where $\mathbf{x}_{1}=\left[x_{1}, x_{2}, \cdots, x_{L}\right]^{T}, \mathbf{x}_{2}=\left[x_{2}, x_{3}, \cdots, x_{L+1}\right]^{T}$ and $\mathbf{x}_{K}=\left[x_{K}, x_{K+1}, \cdots, x_{N}\right]^{T}$ and $(.)^{T}$ denotes the transpose operator. The length of each vector is called the window length, $L$, which can range between $[2, N-1]$. For a time series of length $N$, the total number of lagged vectors is $K=N-L+1$. Following embedding, SVD is performed on the covariance matrix $\mathbf{S}=\mathbf{X X}^{T}$. The SVD operation enables the trajectory matrix $\mathbf{X}$ to be represented in terms of its $L$ elementary matrices: $\mathbf{X}=\mathbf{X}_{1}+\mathbf{X}_{2}+\cdots+\mathbf{X}_{L}$, where $\mathbf{X}_{\mathbf{i}}=\sqrt{\lambda_{i}} \mathbf{u}_{i} \mathbf{v}_{i}^{T}, \lambda_{i}$ is the $i^{\text {th }}$ eigenvalue, and $\mathbf{u}_{i}$ and $\mathbf{v}_{i}$ are $i^{\text {th }}$ left and right eigenvectors, respectively.

2) Reconstruction: This step comprises eigentriple grouping and diagonal averaging to reconstruct elementary matrices $\mathbf{X}_{i}$. First, the trajectory matrix for the desired signal is constructed from one or more elementary matrices. The SVD splits the time series into several groups $I=\left(i_{1}, i_{2}, \cdots, i_{g}\right)$ with $g$ number of eigenvalues. The resultant trajectory matrix of the desired signal is,

$$
\hat{\mathbf{X}}_{I}=\sum_{j=i_{1}}^{i_{g}} \mathbf{X}_{j}=\left[\begin{array}{ccccc}
\hat{x}_{11} & \hat{x}_{12} & \hat{x}_{13} & \ldots & \hat{x}_{1 K} \\
\hat{x}_{21} & \hat{x}_{22} & \hat{x}_{23} & \ldots & \hat{x}_{2 K} \\
\hat{x}_{31} & \hat{x}_{32} & \hat{x}_{33} & \ldots & \hat{x}_{3 K} \\
\vdots & \vdots & \vdots & \vdots & \vdots \\
\hat{x}_{(L-1) 1} & \hat{x}_{(L-1) 2} & \hat{x}_{(L-1) 3} & \ldots & \hat{x}_{(L-1) K} \\
\hat{x}_{L 1} & \hat{x}_{L 2} & \hat{x}_{L 3} & \ldots & \hat{x}_{L K}
\end{array}\right]_{L \times K}
$$

The second step is diagonal averaging. Unlike $\mathbf{X}$, the matrix $\hat{\mathbf{X}}_{I}$ is not a Hankel matrix. Hence, the anti-diagonal elements of $\hat{\mathbf{X}}_{I}$ are averaged to obtain an accurate estimation of each sample. The reconstructed desired series is expressed as $\hat{\mathbf{x}}_{N}=$ $\left[\hat{x}_{1}, \hat{x}_{2}, \hat{x}_{3}, \cdots, \hat{x}_{N}\right]$ where,

$$
\begin{aligned}
\hat{x}_{1} & =\hat{x}_{11} \\
\hat{x}_{2} & =\left(\hat{x}_{12}+\hat{x}_{21}\right) / 2 \\
\vdots & \\
\hat{x}_{L-1} & =\left(\hat{x}_{(L-1) 1}+\hat{x}_{(L-2) 2}+\cdots+\hat{x}_{1(L-1)}\right) /(L-1) \\
\hat{x}_{L} & =\left(\hat{x}_{L 1}+\hat{x}_{(L-1) 2}+\cdots+\hat{x}_{1 L}\right) / L \\
\vdots & \\
\hat{x}_{K} & =\left(\hat{x}_{L(N-2 L+2)}+\hat{x}_{(L-1)(N-2 L+3)}+\cdots+\hat{x}_{1 K}\right) / L \\
\hat{x}_{K+1} & =\left(\hat{x}_{L(N-2 L+3)}+\hat{x}_{(L-1)(N-2 L+4)}+\cdots+\hat{x}_{2 K}\right) /(L-1) \\
\vdots & \\
\hat{x}_{N-1} & =\left(\hat{x}_{L(K-1)}+\hat{x}_{(L-1) K}\right) / 2 \\
\hat{x}_{N} & =\hat{x}_{L K}
\end{aligned}
$$

Any sample can be estimated accurately only when $L$ number of anti-diagonal elements are averaged. For example, $\hat{x}_{L-1}$ and $\hat{x}_{K+1}$ are calculated by averaging $L-1$ elements and are less accurate compared to their adjacent samples $\hat{x}_{L}$ and $\hat{x}_{K}$, respectively. The accuracy reduces as the number of anti-diagonal elements representing the same instant decreases, hence $\hat{x}_{1}$ and $\hat{x}_{N}$ are the most erroneous samples. These $L-1$ samples at the two ends form the transition phase of SSA and are discarded in batch filtering. The rest of the $N-2 L-2$ samples are accurate and possess zero-phase characteristics.

\section{B. Extreme Learning Machine}

For the prediction of voluntary motion, we adopted the ELM algorithm as it can learn the underlying relationship between an input and an output vector in a random feature space efficiently. The learning of the ELM algorithm is 1000 
times faster than the state-of-the-art algorithms such as support vector machines, with comparable or even better generalisation performance [35], [37]. Hence it has been frequently employed in a wide range of industrial applications [38]-[41]. Briefly, if a training set includes $N$ distinct input, $\mathbf{x}_{i} \mid \in \mathbb{R}^{m}$, and target, $\mathbf{t}_{i} \mid \in \mathbb{R}^{n}$, vectors, the ELM algorithm performs a nonlinear mapping as,

$$
f_{\tilde{N}}\left(\mathbf{x}_{j}\right)=\sum_{i=1}^{\tilde{N}} \beta_{i} g_{i}\left(\mathbf{w}_{i} \cdot \mathbf{x}_{j}+b_{i}\right)=\hat{\mathbf{t}}_{j}, \text { for } j=1, \cdots, N
$$

where $\tilde{N}$ is the number of hidden neurons, $\hat{\mathbf{t}}_{j}$ is the predicted target signal, $g_{i}($.$) is the activation function, and$ $\mathbf{w}_{i}=\left[w_{i 1}, w_{i 2}, \cdots, w_{i m}\right]^{T}$ is the output weight vector which links the $i^{t h}$ hidden neuron to input vector $\mathbf{x}$. Further, $\boldsymbol{\beta}_{i}=$ $\left[\beta_{i 1}, \beta_{i 2}, \cdots, \beta_{i n}\right]^{T}$ represents the output weight vector which links the $i^{t h}$ hidden neuron to output vector $\mathbf{t}_{i}$, and $b_{i}$ is the $i^{t h}$ hidden layer bias. The $N$ equations of (1) can be written as $\mathbf{H} \boldsymbol{\beta}=\mathbf{T}$ where $\mathbf{H}$ is a hidden layer output matrix, $\boldsymbol{\beta}$ is the output weight matrix and $\mathbf{T}$ is the target matrix.

\section{Proposed Methods}

\section{A. RSSA: zero-phase estimator}

In batch processing, the covariance matrix formed by $N$ number of samples is decomposed to extract the desired voluntary and tremor signal from the whole motion. To use the SSA algorithm in real-time, when the $(N+1)^{t h}$ sample arrives, the covariance matrix is amended to form the weighted covariance matrix, $\mathbf{S}_{\mathbf{w}}\left(N^{\prime}\right)$, which corresponds to the inclusion of an additional column namely the $(K+1)^{\text {th }}$ lagged vector [42]. Therefore when the $(N+i)^{t h}$ sample arrives, $\mathbf{S}_{\mathbf{w}}\left(N^{\prime}\right)$ is updated as below [42],

$$
\mathbf{S}_{\mathbf{w}}\left(N^{\prime}\right)=(1-\gamma) \mathbf{S}_{\mathbf{w}}\left(N^{\prime}-1\right)+\gamma \mathbf{x}_{K^{\prime}} \mathbf{x}_{K^{\prime}}^{T}
$$

where $N^{\prime}=N+i, K^{\prime}=K+i, \gamma=1 / N^{\prime}$ is a forgetting factor and $\mathbf{x}_{K^{\prime}}$ is the last column of the trajectory matrix $\mathbf{X}$. After eigentriple grouping, the reconstructed matrix will be:

$$
\hat{\mathbf{X}}_{I}=\left[\begin{array}{cccc|c}
\hat{x}_{11} & \hat{x}_{12} & \hat{x}_{13} & \ldots & \hat{x}_{1 K^{\prime}} \\
\hat{x}_{21} & \hat{x}_{22} & \hat{x}_{23} & \ldots & \hat{x}_{2 K^{\prime}} \\
\hat{x}_{31} & \hat{x}_{32} & \hat{x}_{33} & \ldots & \hat{x}_{3 K^{\prime}} \\
\vdots & \vdots & \vdots & \vdots & \vdots \\
\hat{x}_{(L-1) 1} & \hat{x}_{(L-1) 2} & \hat{x}_{(L-1) 3} & \ldots & \hat{x}_{(L-1) K^{\prime}} \\
\hat{x}_{L 1} & \hat{x}_{L 2} & \hat{x}_{L 3} & \ldots & \hat{x}_{L K^{\prime}}
\end{array}\right]_{L \times K^{\prime}}
$$

At this instance, addition of the $K^{\prime}$ th column in the reconstructed matrix $\hat{\mathbf{X}}_{I}$ only contributes to the accuracy of the last $L$ samples from $\hat{x}_{K^{\prime}}$ to $\hat{x}_{N^{\prime}}$. However, the last $L-1$ samples from $\hat{x}_{K^{\prime}+1}$ to $\hat{x}_{N^{\prime}}$ are in a transition phase; the latest sample, which can be estimated accurately with diagonal averaging at this instance, is the $K^{\prime}$ th sample, $\hat{x}_{K^{\prime}}$. Hence to obtain zerophase filtered characteristics, we apply the RSSA algorithm with an $L-1$ sample lag and estimate the $K^{\prime t h}$ sample at each iteration.

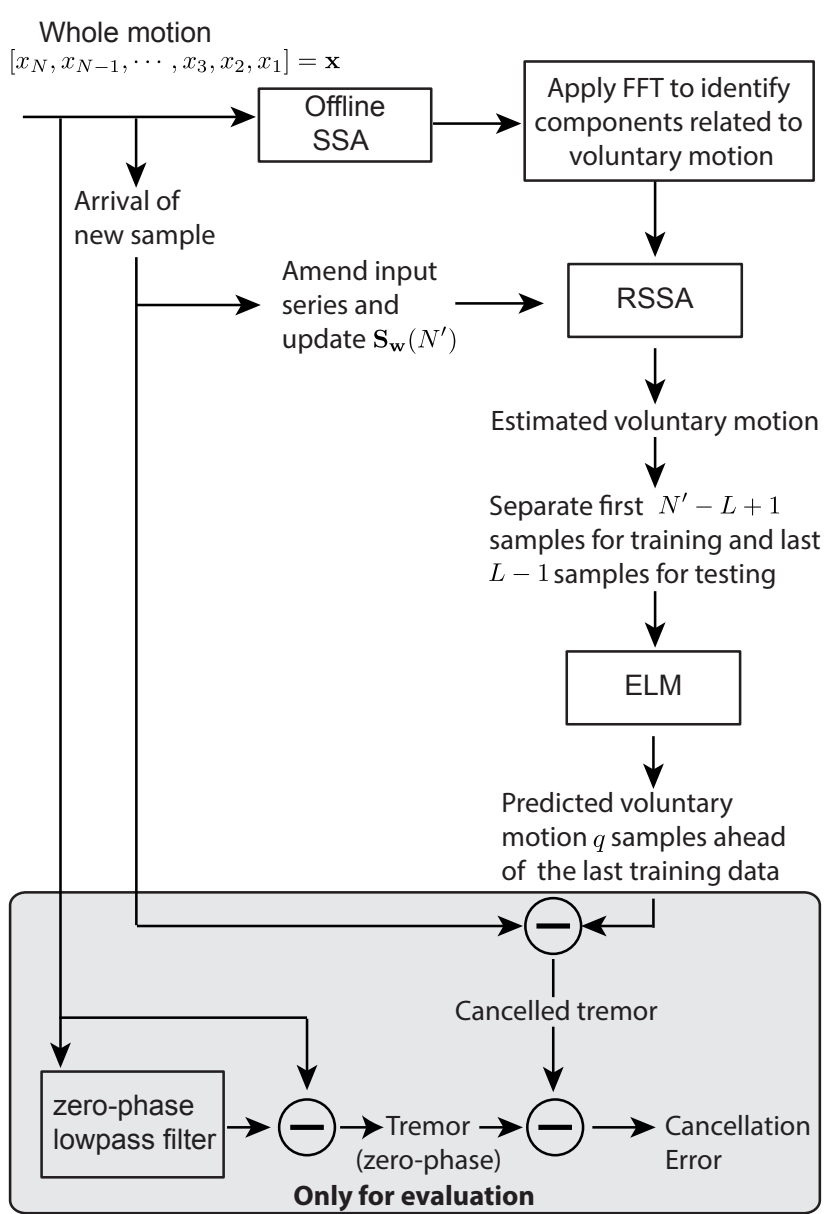

Fig. 2. Functional block diagram of the proposed RSSA-ELM algorithm. RSSA estimates $N^{\prime}$ samples of the voluntary motion, of which the first $N^{\prime}-L+$ 1 are accurate and the last $L-1$ are in the transient phase. The ELM algorithm predicts $q$ samples ahead to shorten the transient phase. The approximated voluntary motion is deducted from the whole motion to obtain the tremor.

\section{B. RSSA-ELM}

The RSSA algorithm decomposes the whole hand motion into dominant voluntary and several oscillatory tremor components. The power of the voluntary motion in the $0-4 \mathrm{~Hz}$ range is significantly larger than that of the tremor components. Initially, we perform an offline SSA on the first $N$ samples to decompose the whole motion. Then, we apply the fast Fourier transform (FFT) on the first few reconstructed components with dominant power, to automatically group all voluntary components below $4 \mathrm{~Hz}$ with dominant power. The use of FFT for automatic grouping of SSA decomposed electroencephalogram signal has been also discussed in [43]. When the $(N+1)^{\text {th }}$ sample arrives, the RSSA algorithm activates to estimate $N+1$ data samples, out of which the last $L-1$ transient samples are corrupted. This way, the RSSA algorithm provides suitable zero-phase type estimation but suffers from $L-1$ sample delay. To minimise this delay, we cascade an ELM predictor with RSSA as shown in Fig. 2. For prediction, the last $L-1$ corrupted samples are segregated for testing and the rest of the initial uncorrupted samples are used for training. While reconstructing the samples at each RSSA iteration, only the last $L$ samples are estimated by averaging. 


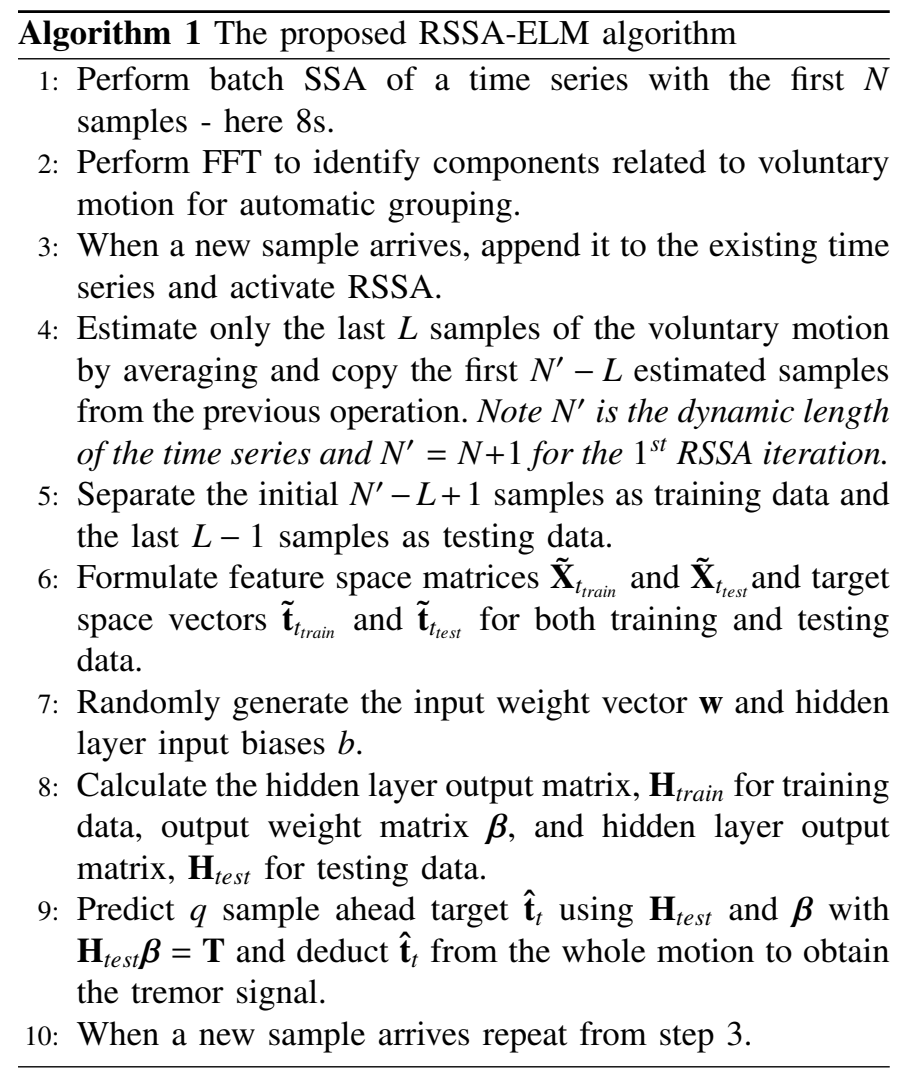

The accuracy of the rest of the initial samples cannot be any further improved by averaging, as we do not get any additional elements representing those initial samples with addition of a new column, as shown in (3). Hence, except for the last $L$ samples, all samples are copied from the previous iteration.

The ELM provides the mapping between input feature space and target space such that each element in target space is $q$ samples ahead of the corresponding input feature space element, that is, $\tilde{\mathbf{t}}_{t}=\tilde{\mathbf{x}}_{t-q}$. The input feature space matrix and target space vector are formulated for both training and testing data. The input feature space matrix is formed by a sequence of delayed target vectors such that $\tilde{\mathbf{X}}_{t}=\left[\tilde{\mathbf{x}}_{t}, \tilde{\mathbf{x}}_{t-1}, \cdots, \tilde{\mathbf{x}}_{t-p}\right]$ where $\tilde{\mathbf{x}}_{t}$ is the vector that is a $q$ sample delayed version of the target space vector $\tilde{\mathbf{t}}_{t}$ and $p$ is the size of the feature space. The output weight matrix obtained by the non-linear mapping implemented in the training phase is used for $q$ sample ahead prediction of the data samples in the testing phase. The steps for the proposed method are described in Algorithm 1.

\section{Two Moving Window RSSA-ELM variants}

To restrict the computational time for each iteration, we propose a method which is a moving window RSSA-ELM, denoted with: MW RSSA-ELM. In the MW RSSA-ELM setting, when the $(N+1)^{t h}$ sample arrives, the $1^{s t}$ sample is discarded and then the $(N+1)^{\text {th }}$ sample is appended to the data series. Similarly, when the $N^{\prime t h}$ sample arrives such that $N^{\prime}=N+i$, the time series is updated as, $\mathbf{x}=\left[x_{i+1}, x_{i+2}, \cdots, x_{N^{\prime}}\right]^{T}$. Hence, the size of $\mathbf{X}$ remains fixed, that is, $(L \times K)$. After application of the SVD and grouping, the resultant trajectory matrix is,

$$
\hat{\mathbf{X}}_{I}=\left[\begin{array}{ccccc}
\hat{x}_{(i+1) 1} & \hat{x}_{(i+1) 2} & \hat{x}_{(i+1) 3} & \ldots & \hat{x}_{(i+1) K} \\
\hat{x}_{(i+2) 1} & \hat{x}_{(i+2) 2} & \hat{x}_{(i+2) 3} & \ldots & \hat{x}_{(i+2) K} \\
\hat{x}_{(i+3) 1} & \hat{x}_{(i+3) 2} & \hat{x}_{(i+3) 3} & \ldots & \hat{x}_{(i+3) K} \\
\vdots & \vdots & \vdots & \vdots & \vdots \\
\hat{x}_{(i+L-1) 1} & \hat{x}_{(i+L-1) 2} & \hat{x}_{(i+L-1) 3} & \ldots & \hat{x}_{(i+L-1) K} \\
\hat{x}_{(i+L) 1} & \hat{x}_{(i+L) 2} & \hat{x}_{(i+L) 3} & \ldots & \hat{x}_{(i+L) K}
\end{array}\right]_{L \times K} .
$$

In the MW RSSA-ELM method, every time RSSA is performed, all the $N$ samples are estimated by diagonal averaging as in the conventional RSSA [42]. Moreover, we developed a second version of the MW RSSA-ELM and called it Enhanced MW RSSA-ELM. In this method, the initial $N-L$ samples, $\hat{x}_{i+1}$ to $\hat{x}_{i+K-1}$, are copied from the previous iteration and only the last $L$ samples, $\hat{x}_{i+K}$ to $\hat{x}_{N^{\prime}}$, are estimated by averaging. By doing so, the first $L-1$ samples are averaged with $L$ antidiagonal elements, when the RSSA window is shifted $L-1$ times. Hence these samples are accurately estimated, and are no longer in the transition phase. This approach not only saves significant computational time but also improves accuracy of the first $L-1$ samples at each iteration simultaneously.

\section{Performance Indices}

The efficacy of the proposed algorithms was evaluated with the root mean square error (RMSE) that quantifies the remaining uncompensated residual tremor after the estimation. For $N$ data samples, assuming $x_{k}$ is the $k^{\text {th }}$ sample of the original data, $\hat{x}_{k}$ is the $k^{\text {th }}$ predicted sample, then the RMSE is defined as,

$$
R M S E=\sqrt{\sum_{k=1}^{N}\left(x_{k}-\hat{x}_{k}\right)^{2} / N}
$$

In microsurgeries, it is crucial to suppress the undesired tremulous vibration below $10 \mu m$, hence $R M S E$ of $10 \mu m$ is set as the required threshold for performance evaluation. RMSE above this level implies failure to achieve the required compensation.

Additionally, we calculated Accuracy (\%) that quantifies the percentage match between the actual and estimated tremor signal and is defined as

$$
\operatorname{Accuracy}(\%)=\frac{R M S(X)-R M S E}{R M S\left(x_{k}\right)} \times 100,
$$

where $R M S(X)$ is the root mean square of the original data series $X$ and is defined as $R M S(X)=\sqrt{\sum_{k=1}^{N} x_{k}^{2} / N}$.

Given the RMSE limit of $10 \mu m$, for this dataset, the required threshold of Accuracy (\%) is $70 \%$, as previously validiated in [32], [33]. Accuracy (\%) obtained below this level signifies failure to achieve the required estimation. Throughout this paper, all reported performance measures are averaged across the $x, y$ and $z$ axes.

\section{Results}

\section{A. Recording of Physiological Tremor Data}

Participants: Five medical students with basic surgical skills (novice subjects) and four experienced surgeons (surgeon 
subjects) were recruited. All the subjects provided informed written consent prior to their participation. The study was approved by the local ethics committee at Nanyang Technological University (NTU) [44].

Measurement: With the aim to develop an intelligent hand-held instrument capable of cancelling the physiological hand tremor during microsurgery, the researchers from the Biorobotics lab at NTU developed the optical micro motion sensing system $\left(\mathrm{M}^{2} \mathrm{~S}^{2}\right)$ [45]. The $\mathrm{M}^{2} \mathrm{~S}^{2}$ was embedded with a pair of orthogonally placed position sensitive detectors (PSDs) as depicted in Fig. 3 in [45]. The PSDs tracked the three dimensional (3-D) displacement of the tip of a microsurgical instrument in $x, y$ and $z$ axes, while the participants carried out some synthetic tasks that mimicked the manoeuvres performed during a real microsurgical procedure. The tasks were carried out in the workspace of $\mathrm{M}^{2} \mathrm{~S}^{2}$, which was a target platform of dimension $10 \times 10 \times 10 \mathrm{~mm}^{3}$ as depicted in Fig. 1 and Fig. 2 in [46]. A stylus which had similar mass and design as a typical surgical forceps was designed with a small white reflector ball attached to its tip as depicted in Fig. 4 and Fig. 5 in [46]. When an IR diode illuminated the workspace, the 3-D position of the tip of the instrument was calculated using the centroid position of the reflected IR rays captured by the PSDs.

As the participants performed the tasks in the $\mathrm{M}^{2} \mathrm{~S}^{2}$ workspace, for their visual feedback, a $19^{\prime \prime}$ flat LCD TV screen was provided. The TV screen was placed $70 \mathrm{~cm}$ away from their seating position. The participants were asked to seat comfortably facing the TV screen and hold the stylus between their index and thumb fingers. They were also instructed to rest their wrist on the small platform of $\mathrm{M}^{2} \mathrm{~S}^{2}$. The data was collected at $250 \mathrm{~Hz}$ with a data acquisition card (PD-MF16-150, United Electronics Industries, USA) with a 16 bits resolution. For more details about the $\mathrm{M}^{2} \mathrm{~S}^{2}$ design and data acquisition, the readers are referred to [44]-[46].

Overall, 54 tremor traces were recorded in 3-D while the participants carried out the two tasks:

- Pointing: The participants were asked to point at the centre of the platform and maintain the position of the tip of the stylus at the set point for 30s. For the visual feedback, two dots were displayed on the screen. One dot was stationary and the other could be moved according to the position of the tip of the stylus. The subjects had to overlap the two dots, to maintain the tool tip at the target position [44]. The pointing task was designed to approximate a typical step carried out in retinal surgery, where a surgeon braces the tool motionless against the sclera for a short duration before inserting it through a targeted area of the sclera to perform precise manipulations inside the eyeball [47].

- Tracing: The participants were asked to trace a synthetic circle of $4 \mathrm{~mm}$ diameter with the speed which was realistic to surgical manipulation as the subjects had surgical knowledge. At the beginning of the task, a white circle of $4 \mathrm{~mm}$ diameter was displayed on the screen, the participants had to rotate the displayed dot on this fixed circle clockwise for 30s [44]. This synthetic task was medically relevant and was designed to simulate the incision and vein tracing manoeuvres [46], [47]. The vein

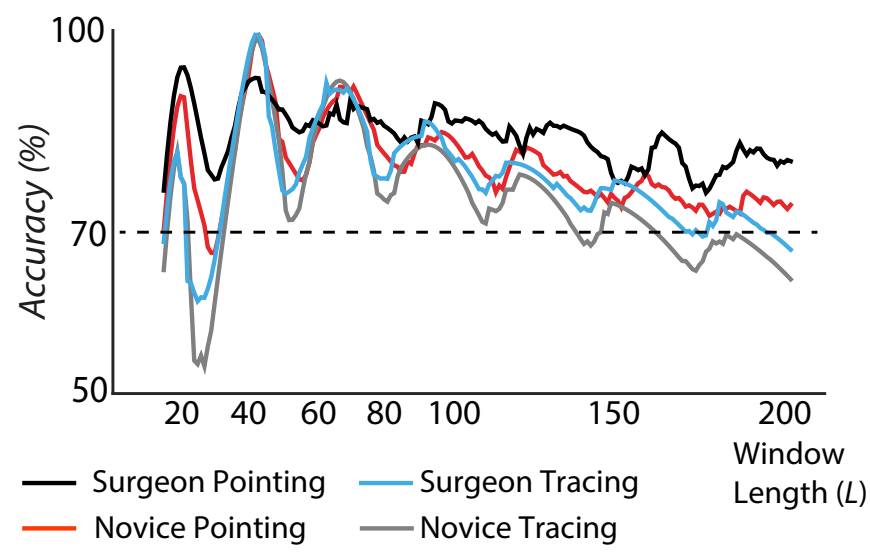

Fig. 3. Accuracy (\%), averaged across all subjects versus $L$. The dashed line shows the minimum acceptable accuracy of $70 \%$.
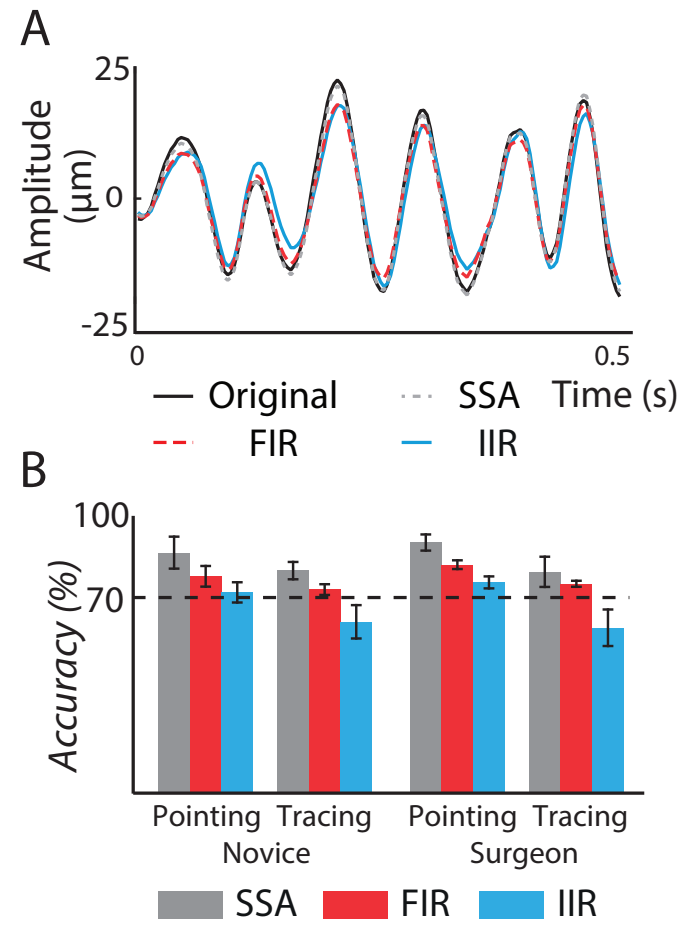

Fig. 4. (A) The SSA filter provides closer approximation of the tremor signal compared to the conventional FIR and IIR filters. (B) Accuracy (\%) averaged across each subject group. The SSA filter outperforms both FIR and IIR filters. The dashed line shows the minimum acceptable accuracy of $70 \%$.

tracing manoeuvre is often performed in a retinal surgery where a surgeon traces curved retinal vein $(\approx 10 \mathrm{~mm})$ choosing a speed comfortable to them [47].

\section{B. The performance of the SSA algorithm - offline analysis}

We applied offline SSA algorithm on the data recorded in the $x y z$ axes independently. To select an optimal window size, we varied $L$ from 15 to 200. Fig. 3 shows Accuracy (\%) averaged across all subjects and the $x y z$ axes. It peaks at certain values of $L$. It has been suggested to separate a desired periodic or quasi-periodic signal from the mixture, $L \geq f_{s} / f_{l}$ [48]; where $f_{s}$ is the sampling frequency and $f_{l}$ is the lowest frequency present in the desired periodic or quasi-periodic 
A. Pointing

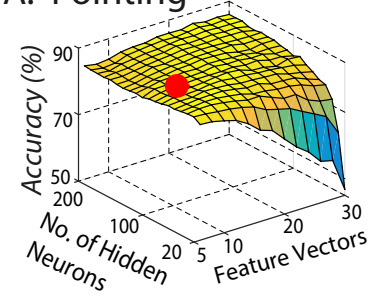

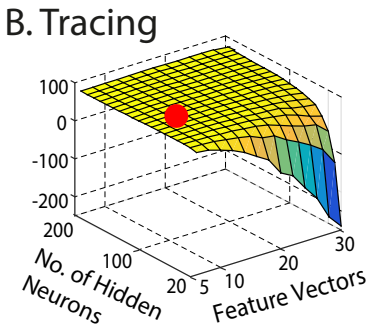

Fig. 5. Accuracy (\%) averaged across all subjects for a range of variation of number of hidden neurons and feature vectors.

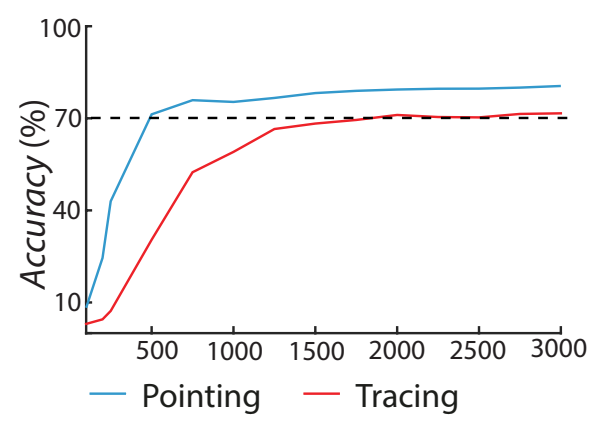

Fig. 6. Prediction Accuracy (\%) of the ELM algorithm against number of training samples, averaged across all subjects.

signal. In our case, the lowest frequency component in the quasi-periodic tremor band is $6 \mathrm{~Hz}$, hence $L$ should be $\geq 42$. We obtained an average Accuracy (\%) of $93.3 \%$ at $L=42$ across all the subjects for both tasks. At $L=20$ where the first peak occurred, the average Accuracy (\%) was $84.3 \%$. The smallest $L$ that can yield the desired accuracy is the optimal selection, as it leads to reduced size of the covariance matrix in the SVD, hence we chose $L=20$.

The performance of the SSA algorithm was compared against conventional FIR and IIR lowpass filters. The SSA algorithm with $L=20$ causes the fixed delay of 19 sample. To choose an FIR filter with similar order, an FIR lowpass filter based on an Hamming window with cut-off frequency $5 \mathrm{~Hz}$ and order 40 was chosen, which causes time delay of 20 sample. The IIR Butterworth filter had $5 \mathrm{~Hz}$ cut-off frequency and was of order 2 . The lower order was chosen to minimize the phase delay which is just 12 samples. The zero-phase filter with the same specifications as the IIR filter was used to extract the original tremor, which served as the ground truth.

Fig. 4(A) shows an $0.5 \mathrm{~s}$ plot of the approximated tremor data in the $z$-axis, obtained from three filters for the novice subject N1. It is evident that the SSA algorithm closely estimates the actual tremor compared to both conventional IIR and FIR filters. Fig. 4(B) shows a bar chart with averaged Accuracy (\%) across each subject group. The SSA algorithm outperforms both FIR and IIR filters in both tasks. The IIR filter fails to achieve the required accuracy for the tracing task. This encouraged adoption of the RSSA-ELM setting for realtime filtering.
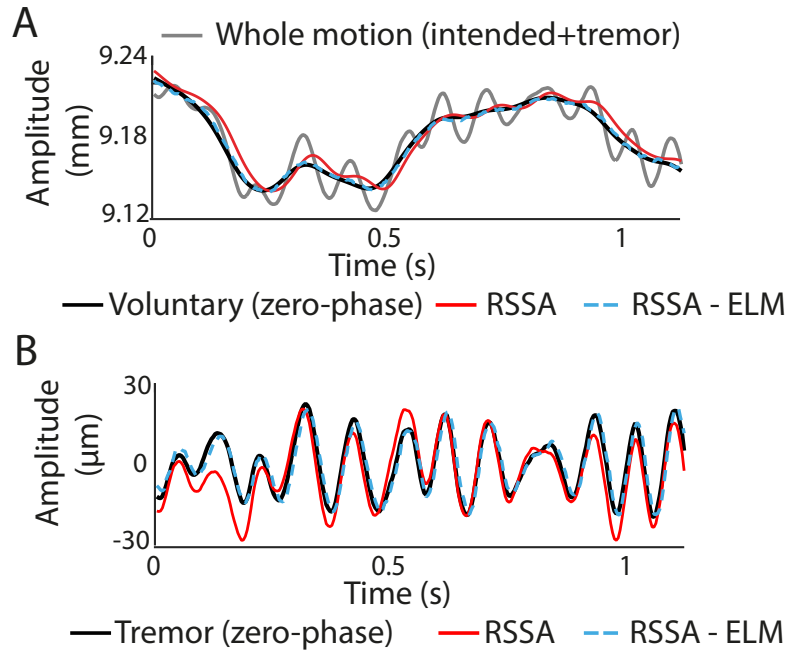

Fig. 7. An example for the performance of the RSSA and RSSA-ELM algorithms in approximating: (A) the intended and (B) tremor components of the whole motion signal.

\section{Selection of ELM Parameters}

Before we proceed with the results of the RSSA-ELM method, we describe how the ELM algorithm was initialised. Performance of ELM depends on the optimum initialisation of the parameters: 1) hidden neurons $\tilde{N}, 2$ ) feature vectors $p$ and 3) the number of offline training samples $N$. We performed a grid search to determine the number of $\tilde{N}$ and p. Taking $80 \%$ of the total data for training and $20 \%$ for testing, the percentage accuracy was calculated for 8-sample ahead prediction. The numbers $\tilde{N}$ and $p$ were varied in the range [20,200] and [6,30], respectively. Fig. 5A and B show Accuracy (\%) averaged across all the subjects for both pointing and tracing tasks. The variation in Accuracy (\%) between two extreme values of $\tilde{N}$ was around $1 \%$ for the pointing task and $2 \%$ for the tracing task. However, between two extreme values of $p$, variation in Accuracy (\%) was significant in both pointing task and tracing task. The optimal parameters of the ELM algorithm were identified as $\tilde{N}=100$ and $p=10$.

In real-time tremor cancellation, it is highly desirable to have the shortest possible processing delay to ensure the faster correspondence and more intuitiveness. In this work, we aim to reduce the RSSA estimation delay by at least half, with the motivation to achieve the required performance within just 9sample (36ms) delay. This delay is similar to the delay caused by the conventional IIR filters. Hence the prediction horizon was set to 10 . The optimal $\tilde{N}$ and $p$ were utilised to compute the minimum size of $N$ and the output of RSSA was fed to the ELM algorithm sample-by-sample as shown in Fig. 2. At each iteration the latest $L-1$ samples were separated for the testing, and the remaining initial samples were used for the training. Training was recursively performed with an arrival of each new sample and the size of the training data was varied from 250 to 3000 samples. Fig. 6 shows Accuracy (\%) averaged across both subject groups for the pointing and tracing tasks. The prediction performance improved significantly up to 750 

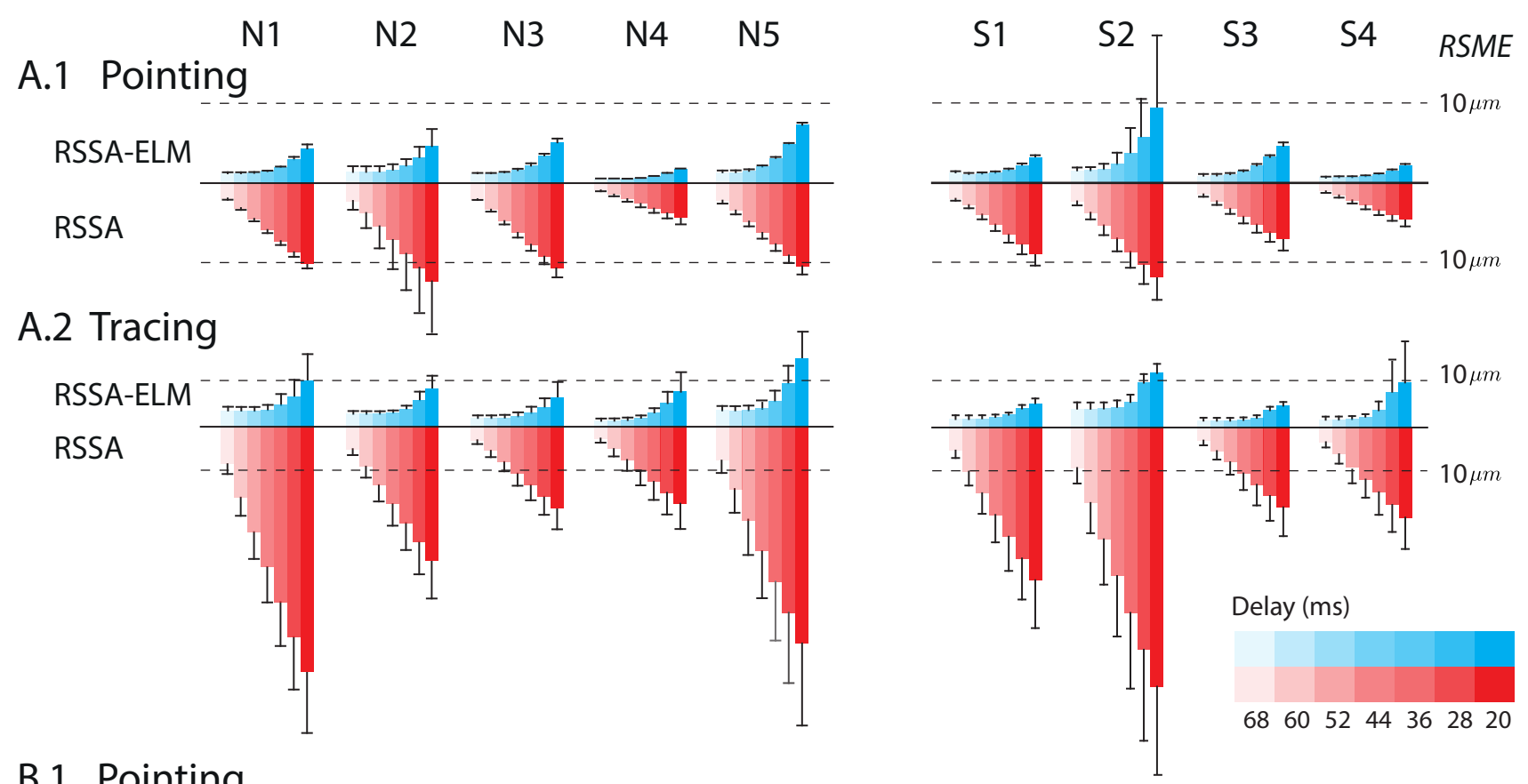

\section{B.1 Pointing}
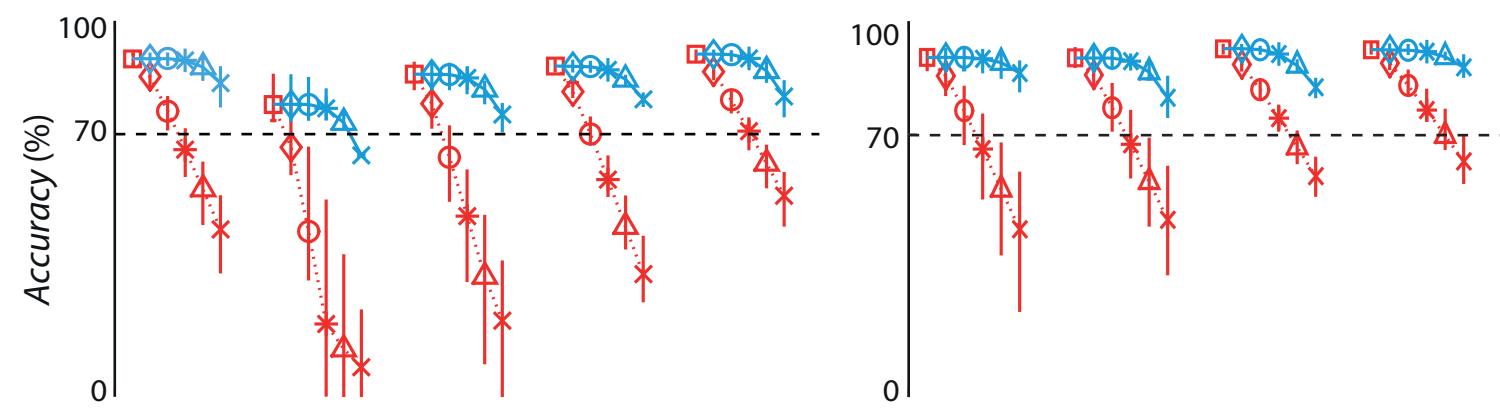

\section{B.2 Tracing}
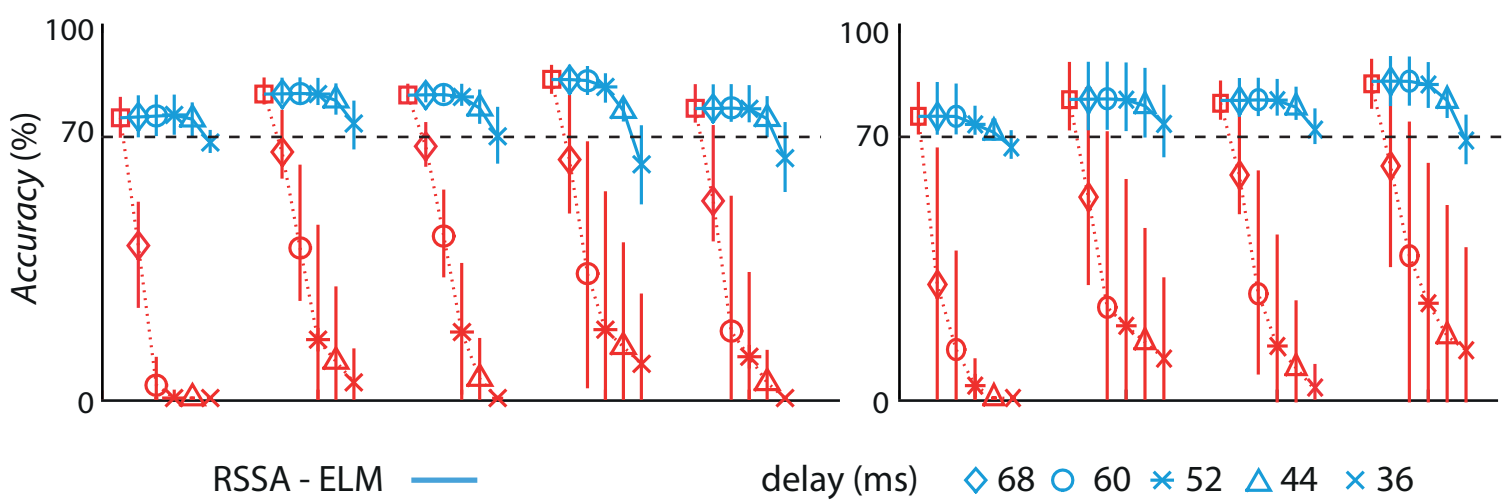

$$
\begin{aligned}
\text { delay (ms) } & \diamond 68 \bigcirc 60 * 52 \Delta 44 \times 36 \\
\square 76 & \diamond 68 \circ 60 * 52 \Delta 44 \times 36
\end{aligned}
$$

RSSA

Fig. 8. Comparison between the RSSA and RSSA-ELM algorithms in terms of RMSE (A) and Accuracy (B); at various delay. The bars beyond the dashed line signify failure to keep the RMSE or Accuracy, below the $10 \mu \mathrm{m}$ or above the $70 \%$ limits, respectively. Results suggest that the RSSA-ELM algorithm needs at least 36ms delay to perform reliably in both tasks and in all subjects. Error bars represent the upper range: [mean, maximum].

samples in the pointing task and 2000 samples in the tracing task. In the tracing task, the prediction accuracy reaches to $70 \%$ only when $N \geq 2000$. Therefore, to achieve the required prediction performance in both tasks, for the majority of traces, the optimum $N$ was set to 2000 (8s).

\section{The performance of the RSSA-ELM - online analysis}

Fig. 7 illustrates a representative performance of the RSSA and RSSA-ELM algorithms on a short excerpt of data, recorded during the pointing task. As it will be described later, for the RSSA algorithm, the estimation was performed at 9- 

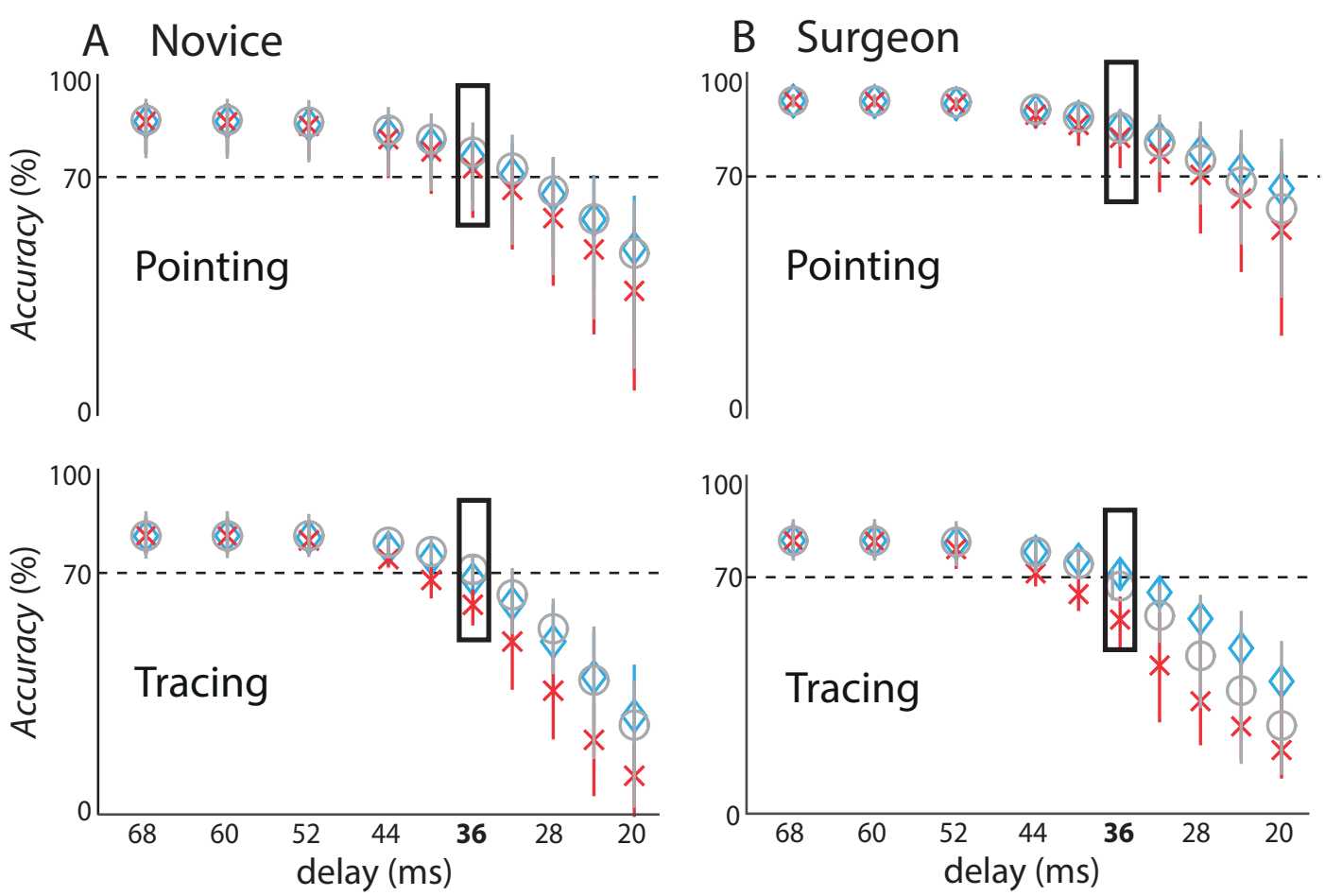

RSSA - ELM $\times$ MW RSSA-ELM

Enhanced MW RSSA-ELM

Fig. 9. Accuracy (\%) obtained with RSSA-ELM, MW RSSA-ELM and Enhanced MW RSSA-ELM, averaged across novice subjects (A) and surgeons (B). Both moving window versions of the RSSA-ELM achieve the same performance as RSSA-ELM but at a significantly reduced computational cost. The black boxes show the performances at $36 \mathrm{~ms}$. Error bars represent the range: [minimum, maximum].

sample delay. Fig. 7A shows that the tremor fluctuations, riding on the voluntary motion, have been effectively attenuated with both the RSSA and RSSA-ELM algorithms. We observe that the RSSA-ELM compensates better for the $36 \mathrm{~ms}$ delay than the RSSA method and closely tracks the intended motion, which was approximated by zero-phase filtering. Similarly, Fig. 7B shows that the RSSA-ELM algorithm tracks the actual tremor signal more successfully.

Fig. 8 shows the RMSE, obtained from both RSSA and RSSA-ELM at various delays. The RSSA-ELM outperforms the RSSA-only algorithm at all time delays, in all participants, in both pointing and tracing tasks. The RMSE of tremor estimation with the RSSA-ELM method is below the $10 \mu \mathrm{m}$ threshold for most subjects even at very short time delays of $20 \mathrm{~ms}$. Most conservatively, when the delay is $36 \mathrm{~ms}$, the RSSAELM limits $R M S E \leq 10 \mu m$ yielding $\geq 70 \%$ Accuracy (\%), meeting both RMSE and Accuracy (\%) requirements for all subjects. The overall performance was better in the pointing task that may reflect its simpler dynamics compared to the tracing task.

\section{E. Moving Window RSSA-ELM}

Fig. 9 shows the plots with Accuracy (\%) obtained from the RSSA-ELM algorithm, averaged across the participants, and that achieved with the two proposed moving window algorithms, namely the MW RSSA-ELM algorithm and the Enhanced MW RSSA-ELM algorithm. For delays as large as
$36 \mathrm{~ms}$, the performance of the RSSA-ELM algorithm matches that of the Enhanced MW RSSA-ELM algorithm. At 36ms lag, the Enhanced MW RSSA-ELM algorithm provides $4 \%$ and 3\% more accuracy than MW RSSA-ELM, for novice and surgeon pointing tasks. Similarly, it provides $10 \%$ better accuracy than the MW RSSA-ELM algorithm for tracing tasks of both subject groups. Beyond 36ms lag, the savings achieved by the MW RSSA-ELM algorithm are at the expense of reduced accuracy. The Enhanced MW RSSA-ELM can recover this reduction only partially.

\section{F. Computational Time}

We quantified the computational time required by the RSSA-ELM, MW RSSA-ELM and the Enhanced MW RSSAELM algorithms as a function of the number of samples, as shown in Fig. 10. Our analysis was run on a PC with an Intel(R) Core i5-4670 CPU (3.40GHz) and 32GB RAM and using Matlab R2017a. In all algorithms the delay was 36ms and the window size was kept to 8s for both moving window algorithms. Crucially, the computational time taken by the RSSA-ELM method increases linearly with the data, whereas the time required by the moving window methods is almost fixed: $20 \mathrm{~ms}$ and 35ms on average for Enhanced MW RSSAELM and MW RSSA-ELM, respectively.

\section{G. Comparision with the existing methods}

Finally, we compared the performance of the proposed Enhanced MW RSSA-ELM against the conventional FIR and IIR 


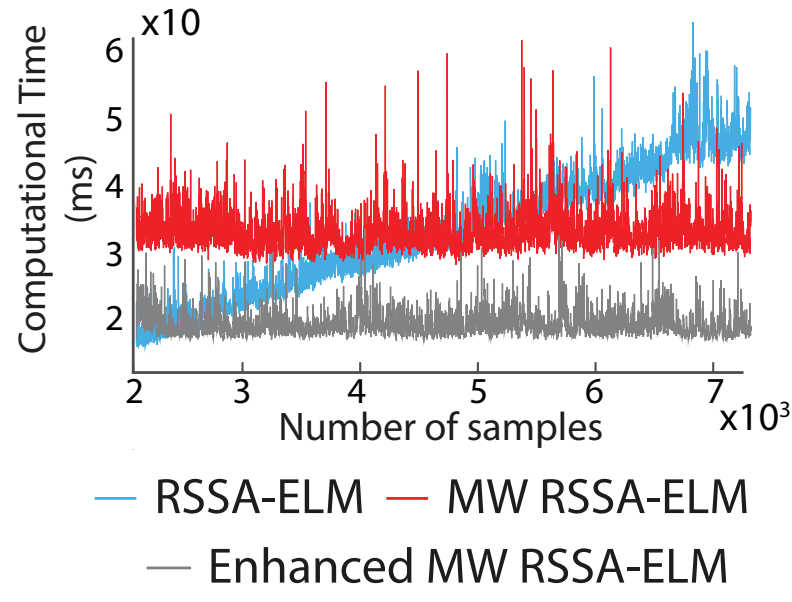

Fig. 10. The required computational time of the RSSA-ELM, MW RSSAELM, and Enhanced MW RSSA-ELM. The Enhanced MW RSSA-ELM achieves the same performance as the RSSA-ELM and MW RSSA-ELM but with a significantly lower computational time.

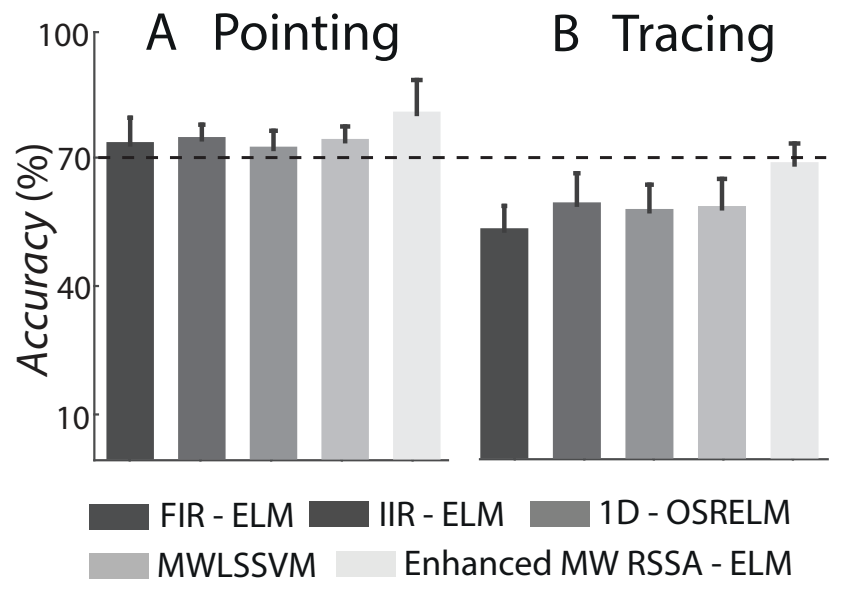

Fig. 11. Accuracy (\%) obtained with the FIR-ELM, IIR-ELM, 1D-OSRELM, MWLSSVM and Enhanced MW RSSA-ELM algorithm, averaged across novice subjects (A) and surgeons (B). The Enhanced MW RSSA-ELM algorithm outperforms all the algorithms. Error bars represent the maximum range.

filters and the existing state-of-the-art MWLSSVM and 1DOSRELM algorithms. A detailed description on the parameters optimisation of MWLSSVM and 1D-OSRELM is provided separately in the supplementary material. For the fairness in evaluation, the ELM predictor cascaded in the Enhanced MW RSSA-ELM to improve the performance, was also appended with the conventional FIR and IIR filters, and are referred as FIR-ELM and IIR-ELM. In FIR-ELM, IIR-ELM and 1DOSRELM algorithms, $N$ was fixed to 2000 samples. Similarly, the moving window length of the Enhanced MW RSSAELM and MWLSSVM was also set to 2000 samples. In all five algorithms, with arrival of every new sample the output weights were updated as detailed in III-B. For the consistency in comparison, the performance of all the algorithms was evaluated at 9-sample delay.

Fig. 11 depicts the averaged compensation Accuracy (\%) obtained for all the subjects for the pointing and tracing tasks, which shows the superior performance of the proposed Enhanced MW RSSA-ELM algorithm compared to the existing methods. The proposed algorithm provided $80.21 \%$ Accuracy $(\%)$ in the pointing task compared to $73.83 \%$ and $72.03 \%$ Accuracy (\%) obtained from the MWLSSVM and the 1DOSRELM algorithm, respectively. Similarly, in the tracing task the Enhanced MW RSSA-ELM algorithm yielded $10 \%$ better Accuracy (\%) compared to the MWLSSVM algorithm and it outperformed all the other algorithms. In an average, over all the subjects and tasks, the Enhanced MW RSSA-ELM algorithm provided $74.54 \%$ Accuracy (\%) which is $8.37 \%$ and $9.61 \%$ higher than the Accuracy (\%) obtained with the MWLSSVM and the 1D-OSRELM algorithm.

\section{Discussion}

The development of an efficient tremor cancellation method that can remove a surgeon's involuntary physiological tremor from the tip of a surgical instrument improves microsurgical performance radically. All of the existing tremor compensating techniques use non-linear phase pre-filtering to isolate the tremor from the whole motion, which significantly distorts the filtered tremor signal and restricts the filtering accuracy. Although, in recent years, the phase correction methods have been proposed, those methods still utilised non-linear phase filters to separate the tremor. Moreover, to rectify the distorted phase, those methods needed zero-phase filtering, which significantly limits their feasibility for real-time implementation. In our preliminary work, we had studied viability of the standard RSSA-only algorithm to filter the tremor signal without causing any phase distortion. In this work, we have applied algorithmic improvement to the standard RSSA algorithm to reduce the computational cost whilst keeping the performance same. We exploited the zero-phase characteristics of this modified RSSA algorithm to develop a combined estimatorpredictor based RSSA-ELM framework to directly extract the voluntary motion and the tremor from the whole motion, without need of any pre-filtering or zero-phase filtering. This is the main distinctiveness of the proposed framework.

We have uniquely formulated the RSSA-ELM structure such that, at every iteration, the last L-1 corrupted samples coming from the RSSA estimator were discarded and only rest of nondistorted samples were fed to the ELM predictor. Thus, the RSSA estimator offered zero-phase type filtering but with the transient delay of 19 sample (76ms). The ELM predictor then learnt the accurate signal fed from the RSSA estimator, and made a forward prediction to shorten this delay to 9 sample (36ms) only. Since the hand tremor is a non-stationary signal and its characteristics varies continuously, this continual feed of the actual tremor signal to the ELM predictor enabled it to constantly track and predict the tremor signal accurately. Therefore, the proposed framework is dynamic, in contrast to the phase correction techniques that could track the tremor signal offline only. The is the major advantage the proposed RSSA-ELM framework.

The cascaded RSSA-ELM framework suppressed the undesired tremulous motion below $10 \mu m$, yielding estimation accuracy over $70 \%$. In a pseudo-real time implementation, it 
can achieve this performance at only 9-sample (36ms) delay. In addition, we proposed two moving window variants of the RSSA-ELM algorithm. These moving window algorithms iteratively operate on a fixed number of samples, so that the computation time is constant regardless of the input data length. We showed that on an average, the Enhanced MW RSSA-ELM algorithm provided $3.5 \%$ and $10 \%$ better performance than MW RSSA-ELM algorithm in the pointing and tracing tasks. The compuational cost of the Enhanced MW RSSA-ELM algorithm is 1.5 times less compared to the MW RSSA-ELM algorithm. The Enhanced MW RSSAELM algorithm outperformed the conventional FIR and IIR filters and the state-of-the-art MWLSSVM and 1D-OSRELM algorithms. As such the proposed framework exceeds the industry standard considerably.

Nonetheless, the proposed framework showed superior performance in a real-time emulation, the extensive experimental testing and verification is crucial for a complete implementation of the proposed framework in a real surgical instrument. Additionally, the extent of compensation entirely depends on manipulator which controls actuation of the tool tip. A full compensation cannot be achieved if the erroneous motion is larger than manipulator range of motion.

Due to the efficient learning rate, the ELM algorithm is gaining popularity in the hardware implementations which require frequent on-chip training [49]-[51]. Therefore, the proposed Enhanced MW RSSA-ELM algorithm is suitable to implement in surgical hand-held tools, such as Micron and its descendant ITrem, for cancellation of tremor in realtime. The RSSA estimator and ELM predictor algorithms were used in this proof-of-concept study to show the potential of the proposed estimation-prediction framework. The proposed framework can be readily adopted to any other suitable estimator which offers zero-phase filtering similar to RSSA; and any other machine learning predictor which provides simple yet efficient learning as ELM.

\section{Conclusions}

We have revisited a long-standing problem in the existing research and offered a novel estimator-predictor based RSSAELM framework which does not require pre-filtering unlike the existing methods. The proposed algorithm can attenuate the undesired tremor below $10 \mu \mathrm{m}$ and yield filtering accuracy over $70 \%$ at only 9 -sample (36ms) delay. For real-time implementation, we proposed two variants of moving window RSSA-ELM algorithm. The Enhanced MW RSSA-ELM algorithm provides $6.75 \%$ more Accuracy (\%) and is 1.5 times faster than the MW RSSA-ELM algorithm. The Enhanced MW RSSA-ELM also outperforms the conventional FIR and IIR filters and beats the performance of the existing state-of-the-art MWLSSVM algorithm by $8.37 \%$.

Our future work includes quaternion extension of the proposed 1-D RSSA-ELM framework for real-time tremor filtering, since the quaternion adaptive filters have shown improved performance compared to their 1-D versions [52]-[54]. We have previously presented quaternion modelling and prediction algorithm for offline tremor filtering in [31], [55], [56].

\section{ACKNOWLEDGMENT}

The authors thank Dr Wei Tech Ang at Nanyang Technological University, Singapore for provision of the data.

The authors would also like to thank two unknown reviewers for their insightful comments and suggestions that led to significantly improve quality of this paper.

\section{REFERENCES}

[1] G. Deuschland, J. Raethjen, M. Lindemann, and P. L. Krack, "The pathophysiology of tremor," Muscle and Nerve, vol. 24, no. 6, pp. 716735, 2001.

[2] C. N. Riviere, R. S. Rader, and N. V. Thakor, "Adaptive canceling of physiological tremor for improved precision in microsurgery," IEEE Trans. Biomed. Eng., vol. 45, no. 7, pp. 839-846, July 1998.

[3] C. N. Riviere, J. Gangloff, and M. D. Mathelin, "Robotic compensation of biological motion to enhance surgical accuracy," Proc. IEEE, vol. 94, no. 9, pp. 1705-1716, Sep. 2006.

[4] Y. S. Kwoh, J. Hou, E. A. Jonckheer, and S. Hayati, "A robot with improved absolute positioning accuracy for CT guided stereotactic brain surgery," IEEE Trans. Biomed. Eng., vol. 35, no. 2, pp. 153-160, Feb. 1988.

[5] I. W. Hunter, T. D. Doukoglou, S. R. Lafontaine, P. G. Charette, L. A. Jones, M. A. Sagar, and G. D. Mallinson, "A teleoperated microsurgical robot and associated virtual environment for eye surgery," Presence: Teleoperators and Virtual Environments, vol. 2, no. 1, pp. 265-280, 1993.

[6] C. N. Riviere and N. V. Thakor, "Modeling and canceling tremor in human-machine interfaces," IEEE Eng. Med. Biol. Mag., vol. 15, no. 3, pp. 29-36, May/June 1996.

[7] W. T. Ang, P. K. Khosla, and C. N. Riviere, "Design of all-accelerometer inertial measurement unit for tremor sensing in hand-held microsurgical instrument," IEEE Int. Conf. Robot Autom., vol. 2, pp. 1781-1786, 2003.

[8] M. Patkin, "Ergonomics applied to the practice of microsurgery," Aust. N. Z. J. Surg., vol. 47, pp. 320-329, 1977.

[9] R. C. Harwell and R. L. Ferguson, "Physiologic tremor and microsurgery," Microsurgery, vol. 4, pp. 187-192, 1983.

[10] S. Charles, "Dexterity enhancement for surgery," in Computer Integrated Surgery: Technology and Clinical Applications (R. H. Taylor, S. Lavallée, G. C. Burdea, and E. R. Mösges, eds.), pp. 467-471, Cambridge, MA: MIT Press, 1996.

[11] W. M. Tang and D. P. Han, "A study of surgical approaches to retinal vascular occlusions," Arch. Ophthalmol., vol. 118, pp. 138-143, 2000.

[12] T. S. Wells, R. A. MacLachlan, and C. N. Riviere, "Toward hybrid position/force control for an active handheld micromanipulator," in Proc. IEEE Int. Conf. Robot. Autom., pp. 772-777, May/June 2014.

[13] Y. N. Aye, S. Zhao, and W. T. Ang, "An enhanced intelligent handheld instrument with visual servo control for 2-dof hand motion error compensation," Int. J. Adv. Robot. Syst., vol. 10, no. 10, p. 355, Jan. 2013.

[14] R. Taylor, J. Funda, B. Eldridge, S. Gomory, K. Gruben, D. LaRose, M. Talamini., L. Kavoussi, and J. Anderson, "A telerobotic assistant for laparoscopic surgery," IEEE Eng. Med. Biol. Mag., vol. 14, no. 3, pp. 279-288, May/June 1995.

[15] G. H. Ballantyne and F. Moll, "The da Vinci telerobotic surgical system: the virtual operative field and telepresence surgery," Surg Clin North Am, vol. 83, no. 6, pp. 1293-1304, 2003.

[16] G. Dogangil, B. L. Davies, and F. R. y Baena, "A review of medical robotics for minimally invasive soft tissue surgery," Proc. Inst. Mech. Eng. H J. Eng. Med., vol. 224, no. 5, pp. 653-679, May 2009.

[17] R. Taylor, P. Jensen, L. Whitcomb, A. Barnes, R. Kumar, D. Stoianovici, P. Gupta, Z. Wang, E. deJuan, and L. Kavoussi, "A steady-hand robotic system for microsurgical augmentation," Int. J. Robot. Res., vol. 18, no. 12, pp. 1201-1210, Dec. 1999.

[18] B. Gonenc, J. Handa, P. Gehlbach, R. H. Taylor, and I. Iordachita, "A comparative study for robot assisted vitreoretinal surgery: Micron vs. the steady-hand robot.," in IEEE Int. Conf. Robot Autom., pp. 4832-4837, 2013.

[19] C. N. Riviere, W. T. Ang, and K. P. Khosla, "Toward active tremor canceling in handheld microsurgical instruments," IEEE Trans. Robot. Autom., vol. 19, no. 5, pp. 793-800, Oct. 2003. 
[20] W. T. Latt, U. X. Tan, C. Y. Shee, C. N. Riviere, and W. T. Ang, "Compact sensing design of a handheld active tremor compensation instrument," IEEE Sens. J., vol. 9, no. 12, pp. 1864-1871, Dec. 2009.

[21] C. Bergeles and G.-Z. Yang, "From passive tool holders to microsurgeons: safer, smaller, smarter surgical robots," IEEE Trans. Biomed. Eng., vol. 61, no. 5, pp. 1565-76, May 2014.

[22] K. J. Craik, "Theory of the human operator in control systems; the operator as an engineering system," Br. J. Psychol. Gen. Sect., vol. 38, pp. 56-61, Dec. 1947.

[23] P. Riley and M. Rosen, "Evaluating manual control devices for those with tremor disability," J. Rehabil. Res. Dev., vol. 24, no. 2, pp. 99-110, Jan. 1987.

[24] M. Javidan, J. Elek, and A. Prochazka, "Attenuation of pathological tremors by functional electrical stimulation II: clinical evaluation," Ann. Biomed. Eng., vol. 20, no. 2, pp. 225-236, 1992.

[25] A. V. Oppenheim and R. W. Schaefer, Discrete-Time Signal Processing. Prentice-Hall, Bergen Country, NJ, USA, 2001.

[26] C. N. Riviere, R. S. Rader, and P. K. Khosla, "Characteristics of hand motion of eye surgeons," in Proc. 19th Annu. Int. Conf. IEEE Eng. Med. Biol. Soc., pp. 1690-1693, Nov. 1997.

[27] S. Tatinati, K. C. Veluvolu, S. Hong, W. T. Latt, and W. T. Ang, "Physiological tremor estimation with autoregressive (AR) model and Kalman filter for robotics applications," IEEE Sens. J., vol. 13, no. 12, pp. 4977-4985, Dec. 2013.

[28] K. C. Veluvolu, W. T. Latt, and W. T. Ang, "Double adaptive bandlimited multiple Fourier linear combiner for real-time estimation/filtering of physiological tremor," Biomed. Sig. Process. Cont., vol. 5, no. 1, pp. 37 44, 2010.

[29] K. C. Veluvolu and W. T. Ang, "Estimation of physiological tremor from accelerometers for real-time applications," Sensors, vol. 11, pp. 30203036, Mar. 2011.

[30] K. C. Veluvolu, S. Tatinati, S. Hong, and W. T. Ang, "Multistep prediction of physiological tremor for surgical robotics applications," IEEE Trans. Biomed. Eng., vol. 60, no. 11, pp. 3074-3082, Nov. 2013.

[31] K. Adhikari, S. Tatinati, W. T. Ang, K. C. Veluvolu, and K. Nazarpour, "A quaternion weighted Fourier linear combiner for modeling physiological tremor," IEEE Trans. Biomed. Eng., vol. 63, Nov. 2016.

[32] S. Tatinati, K. C. Veluvolu, and W. T. Ang, "Multistep prediction of physiological tremor based on machine learning for robotics assisted microsurgery," IEEE Trans. Cybern., vol. 45, no. 2, pp. 328-339, Feb. 2015.

[33] S. Tatinati, K. Nazarpour, W. T. Ang, and K. C. Veluvolu, "Multidimensional modeling of physiological tremor for active compensation in hand-held surgical robotics," IEEE Trans. Ind. Electron., vol. 64, no. 2, pp. 1645-1655, Feb. 2017

[34] N. Golyandina and A. Zhigljavsky, Singular Spectrum Analysis for Time Series. Springer, 2013.

[35] G.-B. Huang, H. Zhou, X. Ding, and R. Zhang, "Extreme learning machine for regression and multiclass classification," IEEE Trans. Cybern., vol. 42, no. 2, pp. 513-529, Apr. 2012.

[36] K. Adhikari, S. Tatinati, K. C. Veluvolu, J. A. Chambers, and K. Nazarpour, "Real-time physiological tremor estimation using recursive singular spectrum analysis," in Proc. 39th Annu. Int. Conf. IEEE Eng. Med. Biol. Soc., pp. 3202-3205, July 2017.

[37] G.-B. Huang, Q.-Y. Zhou, and C.-K. Siew, "Extreme learning machine: A new learning scheme of feedforward neural networks," in Proc. IEEE Int. Joint Conf. Neural Netw., pp. 985-990, July 2004

[38] H. Tian and Z. Mao, "An ensemble ELM based on modified AdaBoost.RT algorithm for predicting the temperature of molten steel in ladle furnace," IEEE Trans. Autom. Sci. Eng., vol. 7, no. 1, pp. 73-80, Jan. 2010.

[39] Y. Yu, T. Choi, and C. Hui, "An intelligent quick prediction algorithm with applications in industrial control and loading problems," IEEE Trans. Autom. Sci. Eng., vol. 9, no. 2, pp. 276-287, Apr. 2012

[40] S. B. Sulistyo, D. Wu, W. L. Woo, S. S. Dlay, and B. Gao, "Computational deep intelligence vision sensing for nutrient content estimation in agricultural automation," IEEE Trans. Autom. Sci. Eng., vol. 15, no. 3, pp. 1243-1257, July 2018.

[41] E. Skordilis and R. Moghaddass, "A double hybrid state-space model for real-time sensor-driven monitoring of deteriorating systems," IEEE Trans. Autom. Sci. Eng., pp. 1-16, June 2019.
[42] O. Haavisto, "Detection and analysis of oscillations in a mineral flotation circuit," Cont. Eng. Pract., vol. 18, no. 1, pp. 23-30, Sept. 2010.

[43] S. Enshaeifar, S. Kouchaki, C. C. Took, and S. Sanei, "Quaternion singular spectrum analysis of electroencephalogram with application in sleep analysis," IEEE Trans. Neural Syst. Rehabil. Eng., vol. 24, no. 1, pp. 57-67, 2016.

[44] E. L. M. Su, T. L. Win, W. T. Ang, T. C. Lim, C. L. Teo, and E. Burdet, "Micromanipulation accuracy in pointing and tracing investigated with a contact-free measurement system," in Proc. 31st Annu. Int. Conf. IEEE Eng. Med. Biol. Soc., pp. 3960-3963, Sep. 2009.

[45] T. L. Win, U. X. Tan, C. Y. Shee, and W. T. Ang, "Design and calibration of an optical micro motion sensing system for micromanipulation tasks," in Proc. IEEE Int. Conf. Robot Autom., pp. 3383-3388, Apr. 2007.

[46] T. L. Win, U. X. Tan, K. C. Veluvolu, J. K. D. Lin, C. Y. Shee, S. Ang, and W. T. Ang, "System to assess accuracy of micromanipulation," in Proc. 29th Annu. Int. Conf. IEEE Eng. Med. Biol. Soc., pp. 5743-5746, Aug. 2007.

[47] B. C. Becker, Vision-based control of handheld micromanipulator for robot-assited retinal surgery. $\mathrm{PhD}$ thesis, Johns Hopkins University, 2012.

[48] F. Ghaderi, H. R. Mohseni, and S. Sanei, "Localizing heart sounds in respiratory signals using singular spectrum analysis," IEEE Trans. Biomed. Eng., vol. 58, no. 12, pp. 3360-3367, Dec. 2011.

[49] M. Bataller-Mompeán, J. M. Martínez-Villena, A. Rosado-Muñoz, J. V. Francés-Víllora, J. F. Guerrero-Martínez, M. Wegrzyn, and M. Adamski, "Support tool for the combined software/hardware design of on-chip ELM training for SLFF neural networks," IEEE Trans Ind. Informat., vol. 12, no. 3, pp. 1114-1123, June 2016

[50] E. Yao and A. Basu, "VLSI extreme learning machine: A design space exploration," IEEE Trans. Very Large Scale Integr. (VLSI) Syst., vol. 25, no. 1, pp. 60-74, Jan. 2017.

[51] M. Rasouli, Y. Chen, A. Basu, S. L. Kukreja, and N. V. Thakor, "An extreme learning machine-based neuromorphic tactile sensing system for texture recognition," IEEE Trans. Biomed. Circuits Syst., vol. 12, no. 2, pp. 313-325, Apr. 2018.

[52] C. Cheong Took and D. P. Mandic, "The quaternion LMS algorithm for adaptive filtering of hypercomplex processes," IEEE Trans. Sig. Process. vol. 57, no. 4, pp. 1316-1327, 2009.

[53] C. Cheong Took and D. P. Mandic, "A quaternion widely linear adaptive filter," IEEE Trans. Sig. Process., vol. 58, no. 8, pp. 4427-4431, 2010.

[54] C. Jahanchahi and D. P. Mandic, "A class of quaternion kalman filters," IEEE Trans. on Neural Netw. Learn. Syst., vol. 25, no. 3, pp. 533-544, Mar. 2014.

[55] K. Adhikari, S. Tatinati, K. C. Veluvolu, and K. Nazarpour, "Modeling 3D tremor signals with a quaternion weighted Fourier linear combiner," in Proc. 7th Annu. Int. IEEE/EMBS Conf. on Neural Engineering (NER), pp. 799-802, Apr. 2015.

[56] Y. Wang, S. Tatinati, K. Adhikari, L. Huang, K. Nazarpour, W. T. Ang, and K. C. Veluvolu, "Multi-step prediction of physiological tremor with random quaternion neurons for surgical robotics applications," IEEE Access, vol. 6, pp. 42216-42226, July 2018.

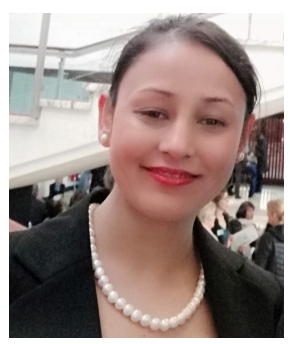

Kabita Adhikari received her B.Eng. degree in Electronics and Communication Engineering from Institute of Engineering, Pulchowk Campus, Tribhuvan University, Nepal in 2004. She received her M.Sc. degree from Northumbria University, Newcastle, UK in 2007 and Ph.D. degree from Newcastle University, Newcastle, UK in 2019. From 2008 to 2010 she worked as a lecturer in South Tyneside College, South Shields, UK. She then joined Newcastle University in 2010 as a Teaching Fellow. Since 2019, she is a lecturer at the School of Engineering, Newcastle University. Her research interests lie primarily in the area of intelligent signal processing, machine learning, preventive and predictive modelling for biomedical applications, AI enabled assistive health-care technologies, and robotics. 


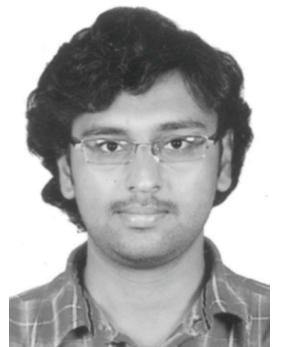

Sivanagaraja Tatinati (Member, IEEE) received the B.Tech. degree in electronics and communication engineering from Acharya Nagarjuna University, Guntur, India, in 2010, and the Ph.D. degree from the School of Electronics Engineering, Kyungpook National University, Daegu, South Korea, in 2016. He held a post-doctoral position at the School of Computer Science, Kyugnpook National University, in 2016. Since 2017, he has been a Research Fellow with the School of Electrical and Electronics Engineering, Nanyang Technological University, Singapore. His current research areas include machine learning, data analytics, natural language processing and generation, and robotics technology for biomedical applications.

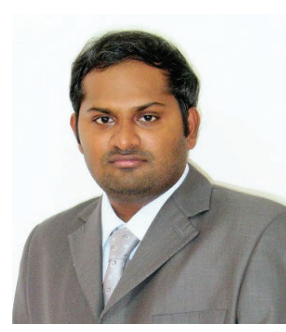

Kalyana C. Veluvolu (Senior Member, IEEE) received the B.Tech. degree in electrical and electronic engineering from Acharya Nagarjuna University, Guntur, India, in 2002, and the Ph.D. degree in electrical engineering from Nanyang Technological University, Singapore, in 2006. From 2006 to 2009, he was a Research Fellow with the Bio-Robotics Group, Robotics Research Center, Nanyang Technological University. Since 2009, he has been with the School of Electronics Engineering, Kyungpook National University, Daegu, South Korea, where he is currently a Professor. He was with the School of Mechanical and Aerospace Engineering, Nanyang Technological University, as a Visiting Professor, from 2016 to 2017. He has also held several visiting positions in University of Newcastle, UK, and University of Valenciennes, France. $\mathrm{He}$ has been a Principal Investigator or a Co-Investigator on a number of research projects funded by government, industry, and universities. He also received several awards for research excellence including the $2020 \mathrm{KNU}$ Academic Award and Excellent Research Award from Ministry of Education, South Korea in 2018. He has authored or coauthored over 130 journal and conference proceedings articles. His current research interests include nonlinear estimation and filtering, sliding mode control, brain-computer interface, autonomous vehicles, biomedical signal processing, and surgical robotics, $\mathrm{He}$ is currently the Associate Editor for Journal of The Franklin Institute, Systems Science and Control Engineering, and Electronics. He has been on technical/program committees of several international conferences.

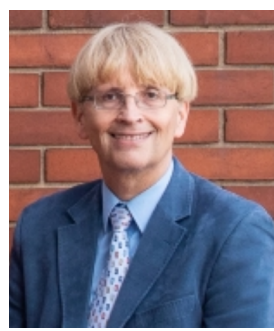

Jonathon A. Chambers (Fellow, IEEE) received the $\mathrm{Ph}$.D. and D.Sc. degrees in signal processing from the Imperial College of Science, Technology and Medicine (Imperial College London), London, U.K., in 1990 and 2014, respectively. From 1991 to 1994, he was a Research Scientist with the Schlumberger Cambridge Research Center, Cambridge, U.K. In 1994, he returned to Imperial College London as a Lecturer in signal processing and was promoted to Reader (Associate Professor), in 1998. From 2001 to 2004, he was the Director of the Center for Digital Signal Processing and a Professor of signal processing with the Division of Engineering, Kings College London. From 2004 to 2007, he was a Cardiff Professorial Research Fellow with the School of Engineering, Cardiff University, Cardiff, U.K. From 2007 to 2014, he led the Advanced Signal Processing Group, School of Electronic, Electrical and Systems Engineering, Loughborough University, where he is currently a Visiting Professor. In 2015, he joined the School of Electrical and Electronic Engineering, Newcastle University, where he was a Professor of signal and information processing and led the ComS2IP Group and is also a Visiting Professor. In 2017, he became the Head of the School of Engineering, University of Leicester. He is also an International Honorary Dean and a Guest Professor with Harbin Engineering University, Harbin, China, with support from the 1000 Talents Scheme. He is a co-author of the books: Recurrent Neural Networks for Prediction: Learning Algorithms, Architectures and Stability (New York, NY, USA: Wiley, 2001) and EEG Signal Processing (New York, NY, USA: Wiley, 2007). He has advised more than 90 researchers through to Ph.D. graduation and has authored/coauthored more than 500 conference papers and journal articles, many of them are in the IEEE journals. His research interests include adaptive signal processing, and machine learning and their applications. He is also a fellow of the Royal Academy of Engineering, U.K., and the Institution of Electrical Engineers. In 2007, he received the first QinetiQ Visiting Fellowship for his outstanding contributions to adaptive signal processing and his contributions to QinetiQ, as a result of his successful industrial collaboration with the international defense systems company QinetiQ. He was the Technical Program Chair of the 15th International Conference on Digital Signal Processing and the 2009 IEEE Workshop on Statistical Signal Processing, both held at Cardiff, U.K., and a Technical Program Co-Chair of the 36th IEEE International Conference on Acoustics, Speech, and Signal Processing, Prague, Czech Republic. He has served on the IEEE Signal Processing Theory and Methods Technical Committee for six years and the IEEE Signal Processing Society Awards Board for three years, together with the Jack Kilby Award Committee. He was an Associate Editor for the IEEE TRANSACTIONS ON SIGNAL PROCESSING for two terms over the periods 1997-1999 and 2004-2007 and as a Senior Area Editor, from 2011 to 2014 . 